\title{
Design of Marine High Power Wireless Charging System
}

\author{
Yajie Chen ${ }^{a}$, Zhiqiang Pan ${ }^{a^{*}}$, Ming $\mathrm{Ni}^{b}$, Haibo Gao ${ }^{c}$, Zhao Pan ${ }^{c}$, He Huang, Yihang Zhu \\ ${ }^{a}$ The 711st Research Institute of China Shipbuilding Industry Corporation, Shanghai, China \\ ${ }^{\mathrm{b}}$ Chongming Communications Committee of Shanghai, Shanghai, China \\ ${ }^{\mathrm{c}}$ School of Energy and Power Engineering, Wuhan University of Technology, Wuhan, China
}

\begin{abstract}
With the application of new energy ships equipped with large-capacity batteries/ultracapacitors in oceans, inland rivers and lakes, the need for high-power wireless charging systems has become increasingly urgent. Based on the analysis of the characteristics of ship charging operation, this paper selected the structure of loosely coupled transformer and introduces its core technology. Then the basic principle of the wireless charging system for the ship is introduced, and the scheme of 1.2 MWwireless charging system is designed according to the specific application. 2.5
\end{abstract}

\section{Introduction}

In recent years, because of its unique advantages in environmental protection, pure electric ship with battery/capacitor pack has become the best way to solve the pollution problems in coastal and inland river ports, and the new energy ship has been developing rapidly. However, the marine charging device is now facing two major problems. Firstly, the power level of the common charging product is too low (only 100-200kW); Secondly, with increasing of the power level and instability of sea condition inport, there are still many interface problems in the wired docking charging system (some high-power plugs can only work for 4000 times).

For the ship, the wired charging technology has the following disadvantages: (1) The charging interface is easy to produce electric spark when charging for the sake of aging. (2) The service life of wired charging device is short. (3) In the case of high-power charging, more than one person is needed to operate, so the working efficiency is low. (4) The metal of the plug head is exposed, so there is a big potential hazard. Wireless charging system can solve the above problems well. It eliminates the need for cable connection between ship and shore to realize safer and more convenient connection and disconnection, also improves operation safety and reduces maintenance cost [1].

In the field of automobiles, GM, Audi, Volvo, Toyota and SAIC have all carried out the research and development of wireless charging facilities. In their sample tests, the maximum charging power can reach $200 \mathrm{~kW}$ and the charging distance is within $300 \mathrm{~mm}$. The BMW i8 is the first to go into mass production, which can be recharged in less than an hour with a Qualcomm Halo $7.2 \mathrm{kw}$ wireless charging system.

Different from the automobile field, the ship floats freely at the dock due to wind, wave, draft and load during loading and unloading, so the system requires high dynamic operating conditions and has fault tolerance for large position changes. The large capacity of marine energy storage system puts forward the demand for highpower charging ${ }^{[2]}$.

WARTSILA cooperated with Convtec to develop the world's first marine wireless charging system, which has been used in the "MF Folgefonn" hybrid ferry running along the coast of Norway. The ferry is 85 meters long, with a gross capacity of 1,182 tons and a recharging power of $1 \mathrm{MW}$. The system is based on inductive power transmission and can be charged wirelessly at a distance of $500 \mathrm{~mm}$. The system and device is shown in Figure 1 and Figure 2.

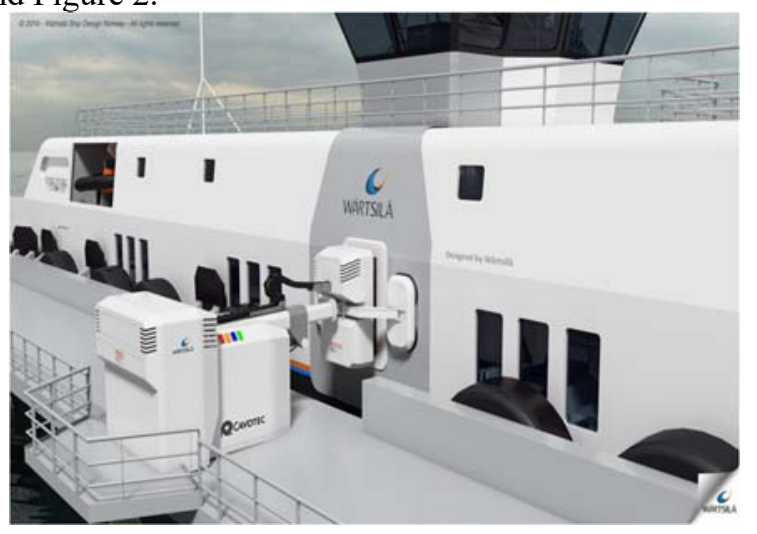

Fig. 1.The world's first marine wireless charging system 


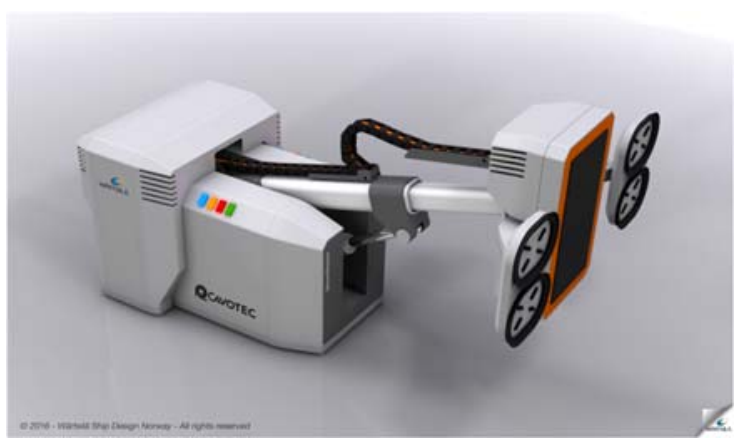

Fig. 2. The marine wireless charging device

In this picture, the land transmitting lateral coil of the system is installed on the hydraulic arm, which will slowly locate the coil according to the changing tide. During charging, the hydraulic arm is kept in a fixed position, and any movement of the ship during operation will be compensated through the control method of the power conversion system.

\section{System working principle}

The wireless charging system of the ship has its own characteristics. Due to the effects of wind and waves on the ship, as well as the changes in tilt and draft caused by loading and unloading, the berthing ship will move relative to the fixed land equipment during loading operation. These movements include ups and downs, sway from side to side, trim/roll, etc. Therefore, the charging system of the ship must be carefully designed to ensure that the power, work efficiency and safety of the system will not be affected by the above movement. The system should have a high tolerance for the relative change of air gap distance and position of the charging contact port, and it should be controlled to automatically compensate for the effect of such relative position change. The Schematic diagram of wireless charging operation of ship is shown in Figure 3 and Figure 4.

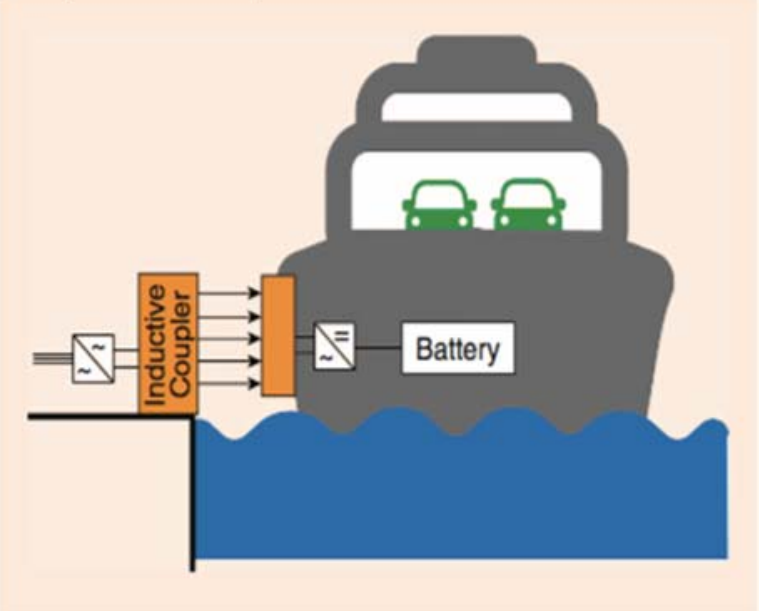

Fig. 3. Schematic diagram of wireless charging operation of ship

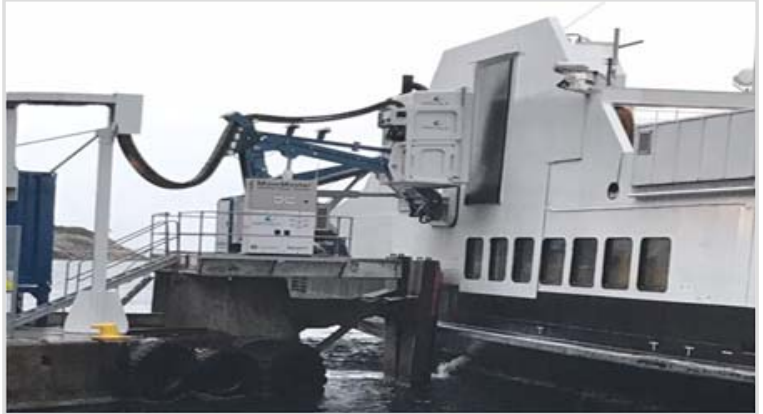

Fig. 4. Physical diagram of wireless charging operation of ship

For the tolerance of large position changes, the wireless charging system can be solved in two ways. Firstly, the coil needs a mechanical positioning system to keep the relative positions of the two coils fixed. Secondly, the tolerance of relative position change should be included in the design and control of the system.

According to the application demand, the wireless charging system hopes to reach the level of wide air gap range, high level dislocation tolerance and high efficiency. The main structure principle of the system is shown in Figure 5.

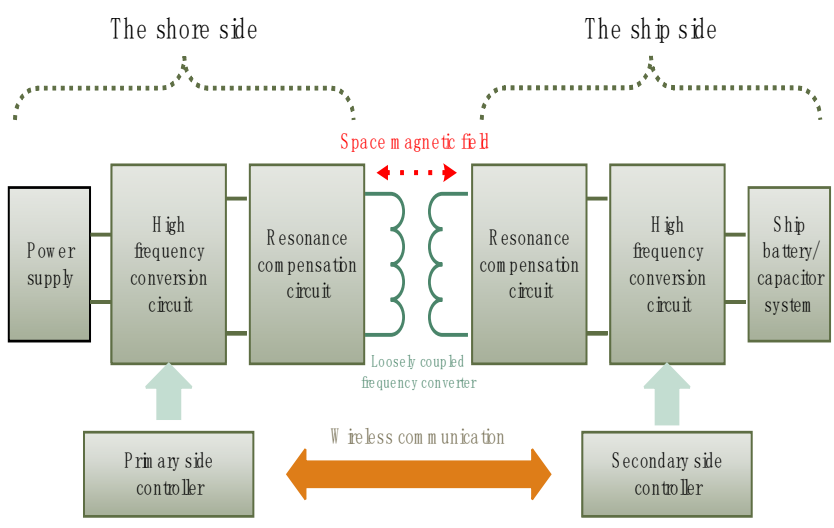

Fig. 5. Schematic diagram of wireless charging system

The working principle of inductively coupled contactless power transmission system is that the transformer is in the state of loose coupling, and the energy is transmitted between the primary and secondary sides through the coupling of space magnetic field. The system is mainly composed of primary side high frequency converter, resonant compensation circuit, coupling transformer coil, secondary side high frequency converter and secondary side resonant compensation circuit. In case of high power, a cooling system for coil cooling and system cooling is also provided.

The resonant compensation circuit uses series-series (SS) compensation, and the SS topology allows the system to be designed based on the standard H-bridge voltage source converter (VSC) topology used to drive power transmission. The SS topology also has the following advantages: the resonant frequency is not affected by the load conditions and is insensitive to changes in the coupling conditions between the two coils [3].

The loosely coupled transformer forms a diverging magnetic field, and the structure of the coil determines the 
direction of the flux path. A reasonable coil design can effectively improve the coupling degree of the primary and secondary edges and the horizontal dislocation tolerance of the transformer on the basis of the original magnetic core.

\section{Main Core Technologies}

\subsection{Structural Design of Loose Coupling Transformer}

The research and application demonstration of noncontact electric energy transmission system has been carried out in the field of automobiles and trains, including long guide rail structure coupling transformer type, disc structure coupling transformer type, DD structure coupling transformer type, electric field and magnetic field double coupling channel electric energy transmission type, and so on. The shapes of the various coupling transformers are shown in Figure 5, Figure 6, Figure 7, Figure 8 , and the performance comparison is shown in Table 1.

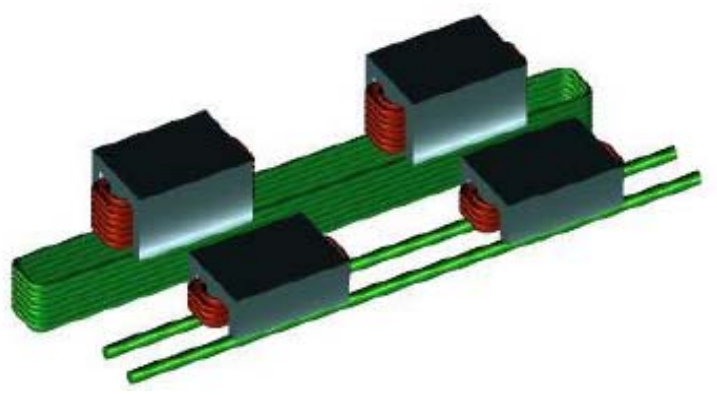

Fig. 5. Long guide rail structure coupling transformer

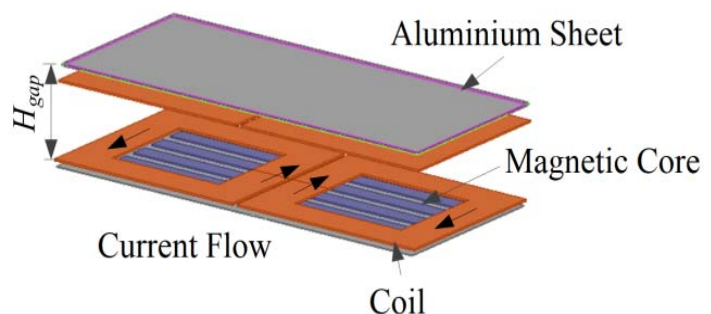

Fig. 6. DD structure coupling transformer

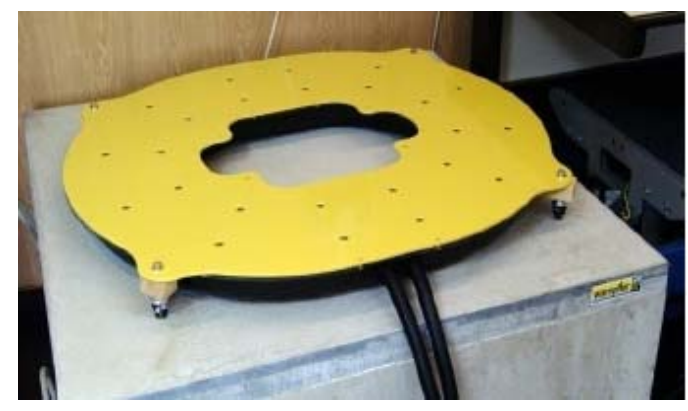

Fig. 7. Disc structure coupling transformer

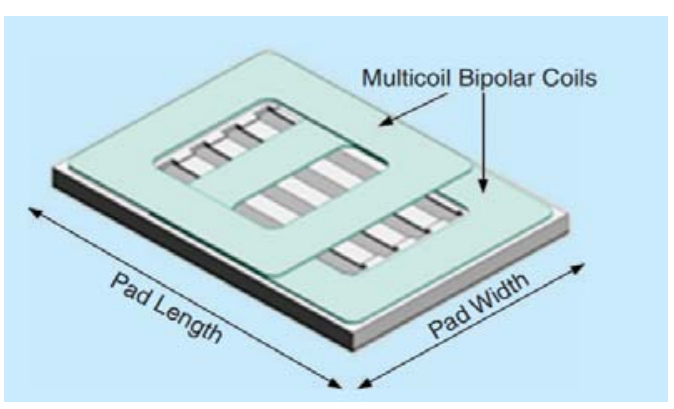

Fig. 8. Bipolar structure coupling transformer

Table 1. Transformer structural characteristics comparison

\begin{tabular}{ccccccc}
\hline Structure & $\begin{array}{c}\text { coupling } \\
\text { coefficient }\end{array}$ & Size & $\begin{array}{c}\text { Structural } \\
\text { cost }\end{array}$ & $\begin{array}{c}\text { Offset fault } \\
\text { tolerance }\end{array}$ & $\begin{array}{c}\text { Circuit } \\
\text { complexity }\end{array}$ & $\begin{array}{c}\text { Electromagnetic } \\
\text { radiation }\end{array}$ \\
\hline Disc shape & low & big & low & low & low & high \\
Square & low & big & low & medium & low & low \\
DD & medium & big & medium & medium & medium & low \\
DDQ & high & medium & high & high & high & low \\
Bipolar & high & medium & high & high & high & low \\
Solenoid & high & small & medium & medium & low & high \\
\hline
\end{tabular}

But the above transformer types have their own advantages and disadvantages.

As the core part of wireless charging system, the design of loose coupling transformer determines the coupling degree of the primary and secondary sides, and the tolerance of the horizontal misalignment of the primary and secondary coils, which directly affects the size of the spatial transmission range of the system ${ }^{[4-5]}$. The loose coupling transformer forms a divergent magnetic field, the coil structure determines the direction of the magnetic flux path, and the optimized design of the transformer coil structure can guide the magnetic flux in the magnetic field, thereby improving the transmission range of the sensing space.

In the field of marine high-power charging, it needs both high offset fault tolerance ability and volume control, and the complexity is medium. Therefore, for transformer design, one point is to consider that the area of the receiving side coil of the ship end is larger than the shore side, and use extended coils; the other point is to adopt different winding structure between the primary and secondary side, which can achieve that under the condition of offset, the primary side and the secondary side winding have complementary coupling performance, 
and always maintaining a high coupling degree; the above two points can improve the offset fault tolerance without increasing too much cost and keeping small size. The basic structure is shown below:

Primary and secondary coils (brown), primary and secondary magnetic cores (black), primary and secondary shields (gray). The Basic Structure of Loose Coupling Transformer is shown in Figure 9.

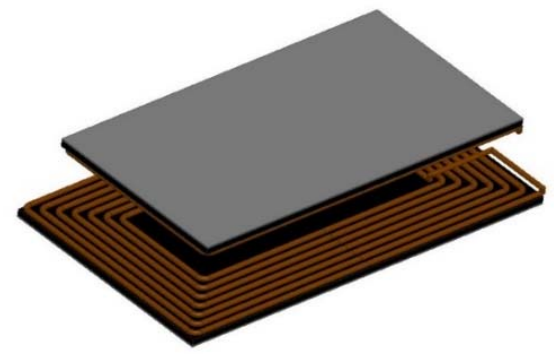

Fig. 9. Basic Structure of Loose Coupling Transformer

The cooling of the high-power loose coupling transformer is worthy of attention. The hollow inside the coil winding uses liquid cooling, and the copper pipe with micro pipe commutation is used; the material structure of the magnetic core is made of nanocrystalline or amorphous material, which is fixedly installed by thermal conductive glue to dissipate the heat. The first set of wireless charging equipment abroad requires 10 minutes of charging and 20 minutes of heat dissipation; in this way, it can charge for 10 minutes and dissipate heat for 10 minutes, which greatly reduces the heat dissipation time.

The area behind the coil can completely shield the magnetic field through the magnetic backplane and the conductive shield, for example, the aluminum plate is used for shielding, the thickness of the aluminum plate has a greater influence on the shielding effectiveness, and the thicker the aluminum plate, the better the shielding effect. Passengers on board will not be exposed to any magnetic field because of the operation of the inductive charger outside the metal casing. On the shore side, a certain safety distance is applied around the transmitting side coil according to the amount of shielding installed on the outside of the hull and the land area around the charger to ensure compliance.

\subsection{Continuous Adaptive Resonance Compensation to Improve Efficiency}

Due to the considerable air gap distance between the transmitting coil and the receiving coil, the inductive power transmission will waste a lot of reactive power compared with the active power that can be transmitted between the coils. The reactive power of the coil is provided by using a resonant network with capacitive compensation on both sides of the charging system to achieve high efficiency and reasonable power electronic conversion period rating. Series-series(ss) compensation is usually preferred for high power applications ${ }^{[6]}$.

Dueto the different load characteristics of inductively coupled power transmission in different operating states (changes in battery charge status, etc.) and the space deviation between the ship and the wireless charging device when ship is docked will cause changes in coupling factors and other uncertain factors and cause the system's working state to change greatly, which making it more demanding on its own parameter adaptability. In order to achieve high-efficiency operation of the system within the working range, the primary side resonance compensation technology is adopted and the zero-phase angle resonance frequency of the output voltage and current of the power supply is controlled to avoid frequency bifurcation and other stability problems, which can effectively improve the transmission performance of the system and reduce the cost of the system ${ }^{[7-8]}$.

\section{System Design}

An all-electric passenger ferry is used to transport vehicles and personnel between the two islands of the Yangtze River in Shanghai. The ship is equipped with $620 \mathrm{KWH}$ super-capacitor, which needs to charge more than 20 times a day. If the wired charging method above MW level is used, the DC cable interface needs to be connected to the socket box on the ferry through the cable winch, using 4400 A, 1000V DC sockets ,2 sockets for DC positive electrode, and 2 sockets for DC negative electrode. The life of the DC socket is 4000 times, that is, about 7 months of use, the DC connector must be replaced. At the same time, two staff members need to be deployed to plug and unplug the charging interface when the ferry is docked. There are many shortcomings, such as personnel fatigue, all-weather open-air operation hazards and inconvenient operation of charging cable.

Faced with such problems, the project team proposed a 1.2 MW wireless charging system scheme, which enhanced the security and service life of charging system. In the scheme, the manipulator is used to track the deviation of the ship when it docked.

The system's input and output power configuration: shore side (input) $950 \mathrm{Vdc}$ bus ( $\pm 5 \%$ ), ship side (output) $950 \mathrm{Vdc}$ bus $(10 \% \sim-8 \%)$. The Maximum transmission power on the ship side is $1200 \mathrm{~kW}$. The rated working transmission efficiency from input side to output side is greater than $90 \%$. When the movement of the ship side is small, the wireless charging system outputs full power. When the ship side moves greatly, the wireless charging system limits the power output.

In this system design, considering that when changing the air gap distance, any change the charging system has the ability of full power transmission in the position corresponding to the confined coupling range, the specific range of tolerable offset is as follows:

1. When the distance between coils is $400 \mathrm{~mm}$ and the deviation along the ship body direction is $\pm 200 \mathrm{~mm}$;

2. When the distance between coils is $400 \mathrm{~mm}$ and the vertical direction of the hull is offset by $\pm 400 \mathrm{~mm}$;

3. When the distance between coils is $400 \pm 70 \mathrm{~mm}$;

4. When the distance between the coils is $400 \mathrm{~mm}$, the deviation is $\pm 150 \mathrm{~mm}$ in the direction of the hull, and the deviation is $\pm 250 \mathrm{~mm}$ in the direction of the vertical hull;

5. When the distance between coils is $400+50 \mathrm{~mm}$, the deviation is $\pm 80 \mathrm{~mm}$ along the hull direction and $\pm 150 \mathrm{~mm}$ perpendicular to the hull. 
The system mainly includes a coil, a shore-side power transmission cabinet, a ship-side power reception cabinet, a robot arm sensor system, and a control system. The primary side converts the $\mathrm{DC}$ bus voltage into $\mathrm{AC}$ to supply power to the primary side coil, and the secondary side coil receives energy and then rectifies it into DC. The system scheme block diagram is shown in Figure 10.

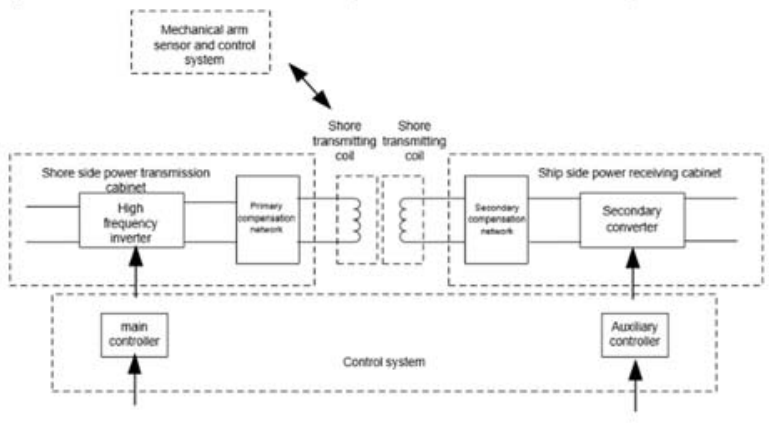

Fig. 10. System scheme block diagram

The coil is the power transmission part of the wireless charging system. It uses electromagnetic coupling to transmit the energy to the receiving coil on the ship side through the shore sending coil, which is composed of winding, magnetic core, shielding and protective shell. Among them, the inner part of the winding part is cooled by liquid; the aluminum plate is used for shielding treatment. Protection grade is IP56. In the design of heat dissipation structure, the coupling simulation of electromagnetic field, temperature field and fluid field is carried out; By optimizing the coil wire distance, the offset tolerance is realized without uniform wire distance.

The original side power transmission cabinet is the core part of the distribution of wireless charging system. It transforms the $\mathrm{DC}$ bus voltage into $\mathrm{AC}$ to supply power to the original side coil. In the process of hull fluctuation, it realizes output voltage stabilization and power transmission, and relevant protection in emergency. The cabinet contains DC circuit breaker, input support capacitor, filter capacitor, DC/AC inverter, resonance capacitor, cooling device, etc.

After receiving the energy from the secondary coil, the ship side power receiving cabinet rectifies it to DC, and realizes the output voltage stabilizing control, offset compatibility control and related protection functions.

The primary side coil is installed on the $\mathrm{X} / \mathrm{Y} / \mathrm{Z}$ threeaxis truss mechanical arm, and the mechanical arm makes flexible follow-up motion, so as to prevent the original secondary side coil from deviating with the water wave motion when carrying out power transmission, resulting in the reduction of transmission efficiency. The manipulator detects the deviations in the three directions of XYZ through the sensor, and calculates the distance and speed to be compensated for the three axes of the manipulator. The controller sends out control signals to control the truss manipulator to realize automatic tracking, so that the deviation of the two coils is kept within a small range.

The control system realizes voltage stabilization and power control during the power transmission process according to the power requirements of the load. The control system can realize the power transmission within a certain range when the original secondary coil has a certain deviation, and when the whole system fails, it can perform fault alarm and protection treatment. The control system provides guarantee for the stable operation and reliability of the overall system. The primary side main controller and the secondary side controller use wireless communication to transmit control signals.

\section{Conclusion}

The battery capacity of new energy ships is about $100 \mathrm{kwh} \sim 7000 \mathrm{kwh}$, the charging demand is often faced with harsh environments such as high temperature, high humidity, high corrosiveness, and heavy load impact. It is required to have fast charging capacity, and the charging power is usually $100 \mathrm{~kW} 2500 \mathrm{kw}$. At present, the maximum supporting power of the fast charging pile is $250 \mathrm{~kW}$, which is too small to meet the needs of the ship. The high-power wireless charging system proposed in this paper can make up for the problems of rigid connection, connector wear and corrosion and inconvenient operation of high-power plug interface of the wired high-power charging system, improve the operation safety and reduce the maintenance cost.

As a non-contact, fast and convenient charging method, it will be the development trend of marine charging in the future, and will have a broad market application prospect.

\section{Acknowledgements}

The authors gratefully acknowledge the financial support provided by Fund of Shang Hai Science and technology Commission(19DZ1203100), r Sund of Ministry of Science and Technology of China (2018YFB1601500), Fund of National Engineering Research Center for Water Transport Safety(A2019002), NSFC-Zhejiang Joint Fund for the Integration of Industrialization and Informatization (U1709215).

\section{References}

1. Wang Xiaoyu. Discussion on wireless charging technology and ship power supply mode [J]. Science and technology and innovation, 2020 (02): 32-34 + 39

2. G. Guidi, J. A. Suul, F. Jenset and I. Sorfonn, "Wireless Charging for Ships: High-Power Inductive Charging for Battery Electric and Plug-In Hybrid Vessels," in IEEE Electrification Magazine, vol. 5, no. 3,pp.2232,Sept.2017,doi:10.1109/MELE.2017.2718 829.

3. G. Guidi and J. A. Suul, "Minimization of converter ratings for MW-scale inductive charger operated under widely variable coupling conditions," 2015 IEEE PELS Workshop on Emerging Technologies: Wireless Power (2015 WoW), Daejeon, 2015, pp. 17, doi: 10.1109/WoW.2015.7132856.

4. Ma Hao, sun Xuan. Design of voltage type coupling electric energy transmission system with primary and 
secondary side series compensation $[\mathrm{J}]$. Chinese Journal of electrical engineering, 2010,30 (15): 48-52

5. Zhou Wenqi, Ma Hao, He Xiangning. Study on different compensation topologies of inductive coupling power transmission system [J]. Journal of electrical technology, 2009,24 (01): 133-139

6. G. Guidi and J. A. Suul, "Minimizing Converter Requirements of Inductive Power Transfer Systems With Constant Voltage Load and Variable Coupling Conditions," in IEEE Transactions on Industrial Electronics, vol. 63, no. 11, pp. 6835-6844, Nov. 2016, doi: 10.1109/TIE.2016.2582459.

7. Zhao Biao, Chen Xiyou, Yu Qingguang. Principle of adaptive resonance technology for non-contact power transmission [J]. New Technology of Electrical Engineering and Energy, 2010, 29 (02): 33-37.

8. Yang Qikun. Design of output control method for non-contact power transmission system based on capacitor array [D]. Chongqing: Master Degree Thesis of Chongqing University, 2012. 